\title{
Pre-hospital delay and emergency medical services in acute myocardial infarction
}

\author{
Seung Hun Lee ${ }^{1,2,{ }^{*}}$, Hyun Kuk Kim ${ }^{3,}$, Myung Ho Jeong ${ }^{1}$, Joo Myung Lee ${ }^{2}$, Hyeon-Cheol Gwon ${ }^{2}$,
} Shung Chull Chae ${ }^{4}$, In-Whan Seong 5 , Jong-Seon Park ${ }^{6}$, Jei Keon Chae, Seung-Ho Hur ${ }^{8}$, Kwang Soo Cha ${ }^{9}$ Hyo-Soo Kim ${ }^{10}$, Ki-Bae Seung ${ }^{11}$, Seung-Woon Rha ${ }^{12}$, Tae Hoon Ahn ${ }^{13}$, Chong-Jin Kim ${ }^{14}$, Jin-Yong Hwang ${ }^{15}$, Dong-Ju Choi ${ }^{16}$, Junghan Yoon ${ }^{17}$, Seung-Jae Joo ${ }^{18}$, Kyung-Kuk Hwang ${ }^{19}$, Doo-Il Kim ${ }^{20}$, Seok Kyu Oh ${ }^{21}$, and for the KAMIR Investigators

\begin{abstract}
${ }^{1}$ Department of Cardiology, Chonnam National University Hospital, Gwangju; ${ }^{2}$ Heart Vascular and Stroke Institute, Samsung Medical Center, Sungkyunkwan University School of Medicine, Seoul; ${ }^{3}$ Department of Cardiology, Chosun University Hospital, Gwangju; ${ }^{4}$ Department of Internal Medicine, Kyungpook National University Hospital, Daegu; ${ }^{5}$ Department of Internal Medicine, Chungnam National University Hospital, Daejeon; ${ }^{6}$ Division of Cardiology, Yeungnam University Medical Center, Daegu; ${ }^{7}$ Division of Cardiology, Department of Internal Medicine, Chonbuk National University Medical School, Jeonju; ${ }^{8}$ Department of Cardiovascular Medicine, Keimyung University Dongsan Medical Center, Daegu; ${ }^{9}$ Department of Cardiology, Pusan National University Hospital, Busan; ${ }^{10}$ Cardiovascular Center, Department of Internal Medicine, Seoul National University Hospital, Seoul; ${ }^{11}$ Division of Cardiology, Department of Internal Medicine, College of Medicine, The Catholic University of Korea, Seoul; ${ }^{12}$ Cardiovascular Center, Korea University Guro Hospital, Seoul; ${ }^{13}$ Department of Cardiology, Gachon University Gil Medical Center, Incheon; ${ }^{14}$ Department of Cardiology, Kyung Hee University Hospital at Gangdong, Seoul; ${ }^{15}$ Department of Internal Medicine, Gyeongsang National University Hospital, Jinju; ${ }^{16}$ Cardiovascular Center, Seoul National University Bundang Hospital, Seongnam; ${ }^{17}$ Division of Cardiology, Department of Internal Medicine, Wonju Severance Christian Hospital, Yonsei University Wonju College of Medicine, Wonju; ${ }^{18}$ Department of Internal Medicine, Jeju National University School of Medicine, Jeju; ${ }^{19}$ Department of Internal Medicine, Chungbuk National University College of Medicine, Cheongju; ${ }^{20}$ Department of Internal Medicine, Inje University Haeundae Paik Hospital, Busan; ${ }^{21}$ Division of Cardiology, Department of Internal Medicine, Wonkwang University School of Medicine, Iksan, Korea
\end{abstract}

Background/Aims: Minimising total ischemic time (TIT) is important for improving clinical outcomes in patients with ST-segment elevation myocardial infarction who have undergone percutaneous coronary intervention (PCI). TIT has not shown a significant improvement due to persistent pre-hospital delay. This study aimed to investigate the risk factors associated with pre-hospital delay.

Methods: Individuals enrolled in the Korea Acute Myocardial Infarction Registry-National Institutes of Health between 2011 and 2015 were included in this study. The study population was analyzed according to the symptomto-door time (STDT; within 60 or $>60$ minutes), and according to the type of hospital visit (emergency medical services [EMS], non-PCI center, or PCI center).

Results: A total of 4,874 patients were included in the analysis, of whom $28.4 \%$ arrived at the hospital within 60 minutes of symptom-onset. Old age ( $>65$ years), female gender, and renewed ischemia were independent predictors of delayed STDT. Utilising EMS was the only factor shown to reduce STDT within 60 minutes, even when cardiogenic shock was evident. The overall frequency of EMS utilisation was low (21.7\%). Female gender was associated with not utilising EMS, whereas cardiogenic shock, previous myocardial infarction, familial history of ischemic heart disease, and off-hour visits were associated with utilising EMS.

Conclusions: Factors associated with delayed STDT and not utilising EMS could be targets for preventive intervention to improve STDT and TIT.

Keywords: Time factors; Emergency medical services; Myocardial infarction; Cardiogenic shock; Prognosis

Received: April 8, 2019

Revised : June 12, 2019

Accepted: June 19, 2019

\section{Correspondence to Myung Ho Jeong, M.D.}

Department of Cardiology, Chonnam National University Hospital, 42 Jebong-ro, Donggu, Gwangju 61469, Korea

Tel: +82-62-220-6243, Fax: +82-62-228-7174, E-mail: myungho@chollian.net

https://orcid.org/0000-0003-4173-1494

*These authors contributed equally to this work. 


\section{INTRODUCTION}

Minimising total ischemic time (TIT) is important for improving the clinical outcome of patients with ST-segment elevation myocardial infarction (STEMI) [1]. Over recent decades, timely reperfusion of the culprit vessel has been a major goal of STEMI treatment. Prompt treatment is required for myocardial salvage because of the time-dependent nature of reperfusion therapies such as primary percutaneous coronary intervention (PPCI) $[2,3]$. Despite the improved door-to-balloon times (DTBTs), no reduction in total ischemic time has been observed [4]. One of the barriers to reducing ischemic time is a pre-hospital (or patient-related) delay, which is usually expressed as symptom-to-door time (STDT) [5].

STDT could be one of the most important factors in reducing TIT in STEMI patients undergoing PPCI. In terms of the healthcare system, regional well-organised emergency medical services (EMS) would be helpful for reducing the time to reperfusion [6]. Nevertheless, in real-world clinical practice, not all patients utilised EMS, which may jeopardise myocardial salvage efforts. Moreover, recognising the patient factors affecting STDT or EMS utilisation is more important if the country or province is relatively small, like Korea, which is highly urbanised and densely populated, making it easier to visit the hospital using private transportation. The present study sought to investigate factors associated with pre-hospital delay and utilisation of EMS among STEMI patients.

\section{METHODS}

\section{Study design and patient population}

The study population were derived from the Korea Acute Myocardial Infarction Registry-National Institutes of Health (KAMIR-NIH). The KAMIR-NIH is a prospective, multi-centre, open, and observational online registry supported by the NIH [7]. A full study flow chart is outlined in Fig. 1. The inclusion criteria for this analysis were as follows: (1) final diagnosis of STEMI; (2) underwent PPCI; and (3) STDT within 12 hours. A total of 4,874 STEMI patients were included in the present study. Patients were categorised into two groups according to STDT, utilising the first quartile of the distribu-

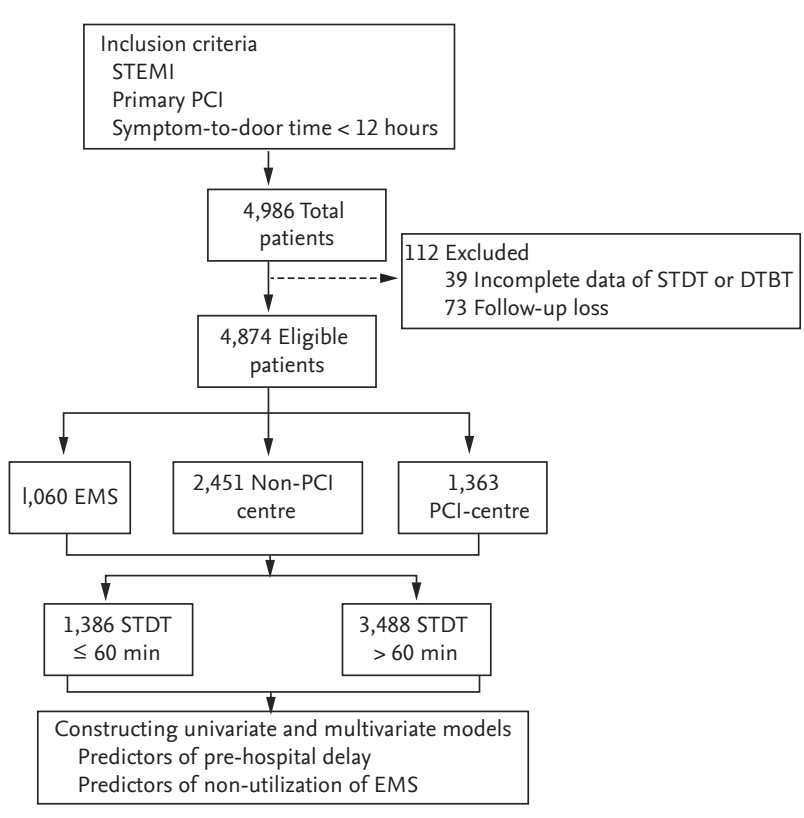

Figure 1. Study flow chart. STEMI, ST-segment elevation myocardial infarction; PCI, percutaneous coronary intervention; STDT, symptom-to-door time; DTBT, door-to-balloon time; EMS, emergency medical services.

tion as a cut-off value (Table 1 and Supplementary Fig. 1 ): $\mathrm{STDT} \leq 60$ minutes and STDT $>60$ minutes.

Symptoms were classified as typical if possible ischemic symptoms included various combinations of chest, upper extremity, mandibular, or epigastric discomfort that were sustained $>20$ minutes [8]. Atypical symptoms included abdominal pain, dyspnoea, weakness, syncope, mental change, nausea, vomiting, and a lack of symptoms. STEMI was diagnosed by a new ST elevation in at least two contiguous leads, measuring > $0.2 \mathrm{mV}$ in leads $\mathrm{V} 1$ to 3 or $0.1 \mathrm{mV}$ in all other leads, or a new left bundle branch block on a 12-lead electrocardiogram and an increase in cardiac markers [8]. Cardiogenic shock was confirmed at the time of admission according to clinical criteria: hypotension (systolic blood pressure $<90 \mathrm{mmHg}$ or catecholamines required to maintain a systolic blood pressure $\geq 90 \mathrm{mmHg}$ ), signs of pulmonary congestion, and impaired end-organ perfusion [9]. Among the type of hospital visits, EMS category was only defined as the patients who visited PCI centre by EMS, PCI-centre category defined as the patients who visited the PCI centre by public or their own transportation, and non-PCI centre category was defined as the 
Table 1. Baseline clinical characteristics

\begin{tabular}{|c|c|c|c|c|c|c|c|}
\hline \multirow[b]{2}{*}{ Characteristic } & \multirow{2}{*}{$\begin{array}{c}\text { Total } \\
(\mathrm{n}=4,874)\end{array}$} & \multicolumn{3}{|c|}{ All patients $(\mathrm{n}=4,874)$} & \multicolumn{3}{|c|}{ Cardiogenic shock $(n=282)$} \\
\hline & & $\begin{array}{c}\mathrm{STDT} \leq 60 \\
\min (\mathrm{n}=1,386)\end{array}$ & $\begin{array}{c}\mathrm{STDT}>60 \\
\min (\mathrm{n}=3,488)\end{array}$ & $p$ value & $\begin{array}{l}\mathrm{STDT} \leq 60 \\
\min (\mathrm{n}=130)\end{array}$ & $\begin{array}{l}\mathrm{STDT}>60 \\
\min (\mathrm{n}=152)\end{array}$ & $p$ value \\
\hline Age, yr & $62.1 \pm 12.6$ & $59.9 \pm 12.3$ & $63.0 \pm 12.7$ & $<0.001$ & $63.0 \pm 12.5$ & $68.3 \pm 13.0$ & 0.001 \\
\hline Age $>65 \mathrm{yr}$ & $1,983(40.7)$ & $458(33.0)$ & $1,525(43 \cdot 7)$ & $<0.001$ & $55(42.3)$ & $94(61.8)$ & 0.002 \\
\hline Male sex & $3,900(80.0)$ & $1,184(85.4)$ & $2,716(77.9)$ & $<0.001$ & $107(82.3)$ & $103(67.8)$ & 0.008 \\
\hline Holiday visits & $1,542(31.6)$ & $449(32.4)$ & $1,093(31.3)$ & 0.494 & $47(36.2)$ & $48(31.6)$ & 0.494 \\
\hline Off-hour visits ${ }^{\mathrm{a}}$ & $1,951(40.0)$ & $597(43.1)$ & $1,354(38.8)$ & 0.007 & $57(43.8)$ & $69(45 \cdot 4)$ & 0.888 \\
\hline Atypical symptoms & $888(18.2)$ & $236(17.0)$ & $652(18.7)$ & 0.008 & $23(17.7)$ & $38(25 \cdot 0)$ & 0.180 \\
\hline Renewed ischemia & $1,012(20.8)$ & $242(17 \cdot 5)$ & $720(22.1)$ & $<0.001$ & $13(10.0)$ & $26(17.1)$ & 0.121 \\
\hline Utilised EMS & $1,060(21.7)$ & $555(44.0)$ & $505(14.5)$ & $<0.001$ & $79(60.8)$ & $41(27.0)$ & $<0.001$ \\
\hline Non-PCI centre & $2,451(50.3)$ & $327(23.6)$ & $2,124(60.9)$ & $<0.001$ & $20(15 \cdot 4)$ & $82(53.9)$ & $<0.001$ \\
\hline STDT, min & $\begin{array}{c}164.4 \pm 146.8 \\
(120[60-220])\end{array}$ & $\begin{array}{c}39 \cdot 3 \pm 16.4 \\
(39[29-56])\end{array}$ & $\begin{array}{c}214.1 \pm 146.0 \\
(169[111-273])\end{array}$ & $<0.001$ & $\begin{array}{l}36.1 \pm 16.8 \\
(35[24-52])\end{array}$ & $\begin{array}{c}205 \cdot 5 \pm 146.5 \\
\left(151\left[105^{-247}\right]\right)\end{array}$ & $<0.001$ \\
\hline DTBT, min & $\begin{array}{l}60.8 \pm 22.9 \\
(58[45-71])\end{array}$ & $\begin{array}{l}62.9 \pm 24.3 \\
(60[46-74])\end{array}$ & $\begin{array}{l}59.9 \pm 22.2 \\
(57[45-70])\end{array}$ & $<0.001$ & $\begin{array}{l}64 \cdot 9 \pm 27 \cdot 3 \\
(61[47-78])\end{array}$ & $\begin{array}{c}64.3 \pm 28.0 \\
(59[46-76])\end{array}$ & 0.865 \\
\hline$\leq 60$ & $2,755(56.5)$ & $719(51.9)$ & $2,036(58.4)$ & $<0.001$ & $63(48.5)$ & $83(54 \cdot 6)$ & 0.363 \\
\hline$\leq 90$ & $4,573(93.8)$ & $1,279(92.3)$ & $3,294(94 \cdot 4)$ & 0.006 & $117(90.0)$ & $135(88.8)$ & 0.898 \\
\hline TIT, min & $\begin{array}{c}225.1 \pm 149.1 \\
(178[120-282])\end{array}$ & $\begin{array}{l}102.2 \pm 29.3 \\
(100[83-118])\end{array}$ & $\begin{array}{c}274 \cdot 0 \pm 149 \cdot 5 \\
(224[167-335])\end{array}$ & $<0.001$ & $\begin{array}{l}101.0 \pm 32.4 \\
(95[80-117])\end{array}$ & $\begin{array}{l}269.8 \pm 152.7 \\
(218[165-313])\end{array}$ & $<0.001$ \\
\hline$\leq 120$ & $1,242(25 \cdot 5)$ & $1,078(77.8)$ & $164(4 \cdot 7)$ & $<0.001$ & $100(76.9)$ & $10(6.6)$ & $<0.001$ \\
\hline$\leq 180$ & $2,487(51.0)$ & $1,358(98.0)$ & $1,129(32.4)$ & $<0.001$ & $125(96.2)$ & $51(33 \cdot 6)$ & $<0.001$ \\
\hline $\mathrm{SBP}, \mathrm{mmHg}$ & $125.1 \pm 32.7$ & $121.3 \pm 36.6$ & $126.6 \pm 30.9$ & $<0.001$ & $55 \cdot 0 \pm 34 \cdot 5$ & $60.1 \pm 30.6$ & 0.197 \\
\hline Heart rate, /min & $75.7 \pm 20.5$ & $73.9 \pm 22.9$ & $76.5 \pm 19.4$ & $<0.001$ & $57.9 \pm 37.0$ & $66.3 \pm 34 \cdot 7$ & 0.051 \\
\hline GRACE score & $123([98-149])$ & $118([94-146])$ & $124([100-151])$ & $<0.001$ & $157([139-177])$ & $163([144-190])$ & 0.020 \\
\hline Cardiogenic shock & $282(5.8)$ & $130(9.4)$ & $152(4 \cdot 4)$ & $<0.001$ & - & - & - \\
\hline Hypertension & $2,229(45 \cdot 7)$ & $637(46.0)$ & $1,592(45.6)$ & 0.866 & $68(52.3)$ & $72(47 \cdot 4)$ & 0.479 \\
\hline Diabetes & $1,160(23.8)$ & $300(21.6)$ & $860(24.7)$ & 0.029 & $35(26.9)$ & $55(36.2)$ & 0.125 \\
\hline Dyslipidaemia & $523(10.7)$ & $169(12.2)$ & $354(10.1)$ & 0.042 & $12(9.2)$ & $13(8.6)$ & 1.000 \\
\hline Smoking & $2,223(45 \cdot 6)$ & $659(47 \cdot 5)$ & $1,564(44.8)$ & 0.093 & $57(43.8)$ & $53(34.9)$ & 0.156 \\
\hline Familial history & $309(6.3)$ & $108(7.8)$ & $201(5.8)$ & 0.011 & $7(5.4)$ & $4(2.6)$ & 0.378 \\
\hline Previous MI & $283(5.8)$ & $97(7.0)$ & $186(5 \cdot 3)$ & 0.140 & $11(8.5)$ & $10(6.6)$ & 0.709 \\
\hline History of stroke & $220(4.5)$ & $53(3.8)$ & $167(4.8)$ & 0.166 & $5(3.8)$ & $7(4 \cdot 6)$ & 0.985 \\
\hline LVEF, \% & $50.7 \pm 10.2$ & $52.4 \pm 9.9$ & $50.0 \pm 10.2$ & $<0.001$ & $50.0 \pm 11.9$ & $43.7 \pm 11.9$ & $<0.001$ \\
\hline CK-MB, ng/mL & $174.0 \pm 185 \cdot 4$ & $157.0 \pm 162.0$ & $180.7 \pm 193.6$ & $<0.001$ & $215.2 \pm 250.9$ & $232.1 \pm 311.5$ & 0.615 \\
\hline Troponin-I, ng/mL & $77 \cdot 7 \pm 127.0$ & $69.6 \pm 152.3$ & $80.9 \pm 115.4$ & 0.023 & $82.9 \pm 166.8$ & $109 \cdot 5 \pm 148.6$ & 0.207 \\
\hline Creatinine, g/dL & $1.0 \pm 0.7$ & $1.1 \pm 0.8$ & $1.0 \pm 0.7$ & 0.049 & $1.2 \pm 0.4$ & $1.3 \pm 0.6$ & 0.008 \\
\hline
\end{tabular}

Values are presented as mean $\pm \mathrm{SD}$, number (\%), or median (interquartile range).

STDT, symptom-to-door time; EMS, emergency medical service; PCI, percutaneous coronary intervention; DTBT, door-toballoon time; TIT, total ischemic time; SBP, systolic blood pressure; GRACE, Global Registry of Acute Coronary Events; MI, myocardial infarction; LVEF, left ventricular ejection fraction; CK-MB, creatine kinase-myocardial band.

${ }^{a}$ Patients who visited hospitals from 6:00 PM to 8:00 AM. 
patients who were transferred from a non-PCI centre to a PCI centre by any mode of transport.

\section{Study variables, outcome measures, and data col- lection}

Baseline characteristics, including demographics, risk factors, and vital signs, were identified at the time of presentation. All patients received evidence-based drugs after establishment of clinical diagnosis [10-12]. PPCI was performed according to the American and European guidelines valid at time of enrolment [10-12], while critical decisions were made at the discretion of the operators. In-hospital mortality and peri-procedural complications were also recorded. All major adverse cardiac events (MACE), including all-cause death, myocardial infarction, or repeat percutaneous coronary intervention (target lesion, target vessel revascularization, or non-target vessel revascularization) were recorded during the 3 years of follow-up. If the patient did not visit the hospital, the outcome data were retrieved from hospital electronic medical records and/or a telephone interview. Attending physicians obtained all clinical data with the assistance of trained clinical research coordinators. The committee of the KAMIR-NIH consisted of healthcare professionals who governed and reviewed all registered data to ensure the adequacy and accuracy of the study. The study protocols were in accordance with the Declaration of Helsinki and were approved by the Institutional Review Board of each participating center (CNUH-2016-075). All patients provided written informed consent.

\section{Statistical analyses}

Discrete or categorical variables were analysed using chi-square or Fisher's exact tests. Continuous variables were analysed using unpaired $t$ tests or Mann-Whitney rank-sum tests according to their distribution. For comparison of multiple groups, the Mantel-Haenszel statistic or analysis of variance were used to test differences according to their distribution. To identify predictors of delayed STDT or not utilising EMS, univariate and multivariate logistic regression analyses were performed. The multivariate model was constructed using all variables with a significance of $p<0.1$ in the univariate analysis, and all relevant variables and their interaction terms could affect pre-hospital delay. A list of candidate variables is presented in Supplementary Table 1. The final multivariate model was then constructed using backward elimination to identify the best Akaike's information criterion; odds ratios (ORs) and 95\% confidence intervals (CIs) were identified. The 3-year cumulative incidence of events was demonstrated by Kaplan-Meier survival curves followed by log-rank or Breslow tests. The Cox proportional hazard model was used to calculate hazard ratios (HRs) and 95\% CIs to compare between-group differences in the cumulative incidence of clinical events. All analyses were two-tailed, and clinical significance was defined as $p<0.05$. Statistical analyses were performed using SPSS 25.0 for windows (IBM Co., Armonk, NY, USA) and R version 3.5.1 (R Foundation for Statistical Computing, Vienna, Austria).

\section{RESULTS}

\section{Baseline clinical characteristics of patients with delayed STDT}

The baseline clinical characteristics of the study population are presented in Table 1 . The total population had a median STDT of 120 minutes (6o to 220 ), median DTBT of 58 minutes ( 45 to 71 ), and median TIT of 178 minutes (120 to 282). The relationship between time intervals in STEMI is shown in Fig. 2. Although DTBT showed a modest correlation with TIT $(r=0.172, p<0.001)$, the most significant correlation was seen between STDT and TIT ( $r=0.989, p<0.001)$. A total of 1,386 patients (28.4\%) visited a hospital within 60 minutes, with a median TIT of 100 minutes ( 83 to 118 ), with $77.8 \%$ patients experiencing a TIT $\leq 120$ minutes. The STDT > 60 minutes group included a higher proportion of older female patients and those who experienced atypical STEMI symptoms at presentation. The incidence of patients who experienced angina prior to presentation (expressed as "renewed ischemia") was higher in STDT $>60$ minutes group. Only $14.5 \%$ of patients with STDT $>60$ minutes utilised EMS and 60.9\% initially visited a non-PCI centre, whereas $44 \%$ of patients who visited within 60 minutes utilised EMS. Patients with STDT $\leq$ 60 minutes presented with a higher incidence of cardiogenic shock. The incidence of dyslipidaemia and familial history of ischemic heart disease (IHD) were lower in the STDT > 60 minutes group, whereas the incidence of 

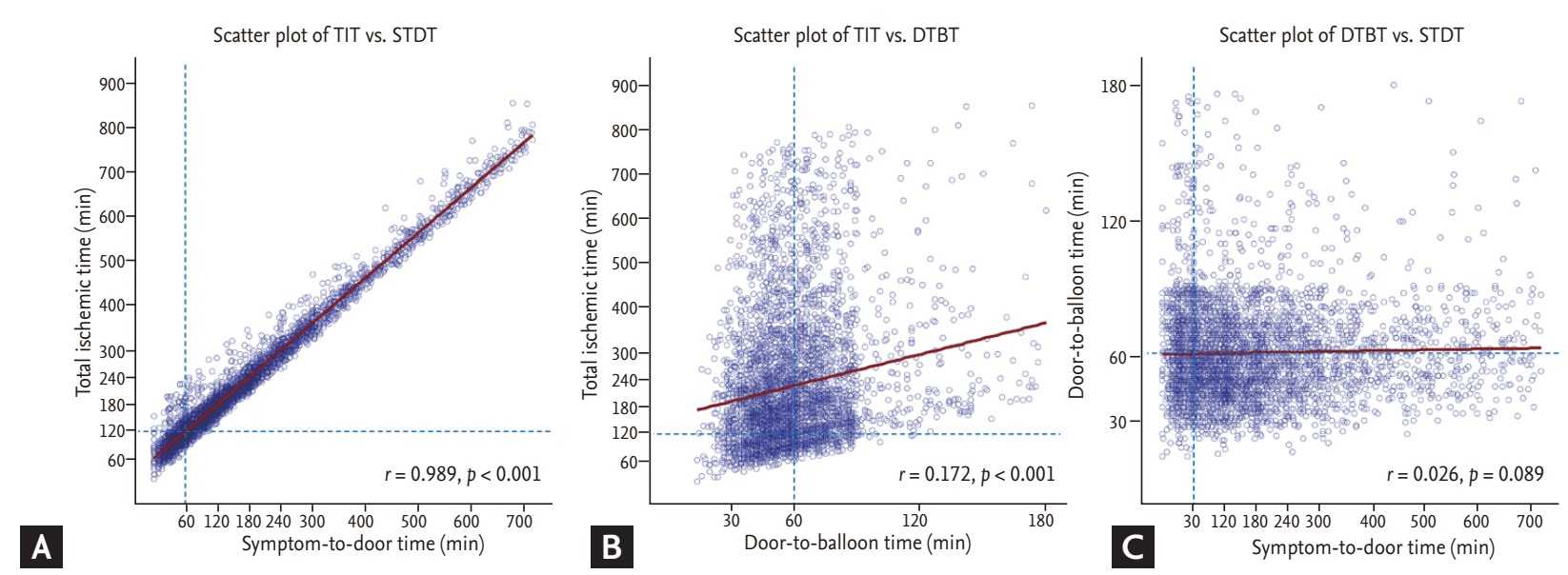

Figure 2. Scatter plots according to time interval. The relationships among time intervals in ST-segment elevation myocardial infarction are shown as scatter plots and linear regression lines: (A) between symptom-to-door time (STDT) and total ischemic time (TIT), (B) door-to-balloon time (DTBT) and TIT, and (C) STDT and DTBT. The STDT and TIT showed a significant correlation $(r=0.989, p<0.001)$.

diabetes was higher in the STDT > 60 minutes group. Peri-procedural echocardiograms showed a lower left ventricular ejection fraction in the STDT $>60$ minutes group. Subgroup analysis, which consisted of patients with cardiogenic shock $(n=282)$, produced results similar to those presented above. However, there were no significant differences in the incidence of diabetes, dyslipidaemia, and a familial history of IHD.

\section{Medical treatments, procedural findings, and in-hospital outcomes}

Detailed data of the medications administered during hospitalisation and the follow-up period are shown in Table 2. There was a difference regarding the use of $\mathrm{P} 2 \mathrm{Y}_{12}$ inhibitors between groups; otherwise, no significant differences were observed. As shown in Table 2, there were no significant differences in treatment procedures based on STDT status, even among patients with cardiogenic shock. After PPCI, the overall survival rate from STEMI was excellent in the total population (4.2\%). The rates of in-hospital mortality and complications did not differ according to the delay in STDT, except for those with ventricular tachyarrhythmia. Among patients with cardiogenic shock, the in-hospital mortality rate was higher in the STDT > 60 minutes group; however, there was no difference in the rate of complications.

\section{Predictors of pre-hospital delay (STDT $>60$ minutes)}

Fig. 3 shows the results of univariate and multivariate logistic regression analysis to identify predictors of delayed STDT. The performance of these logistic regression models was assessed by c-statistics, revealing moderate to good levels of prediction (0.721 for all patients and 0.776 for patients with cardiogenic shock, respectively).

Old age (OR, 1.30; 95\% CI, 1.11 to 1.53; $p<0.001$ ), female gender (OR, 1.27; 95\% CI, 1.05 to 1.54; $p=0.012)$, and renewed ischemia (OR, 1.32; 95\% CI, 1.12 to $1.57 ; p=0.001$ ) were identified as independent risk factors of delayed STDT in all STEMI patients. Although it was not statistically significant, atypical symptoms of old age (OR, 1.43; 95\% CI, 0.99 to $2.05 ; p=0.056$ ) seemed to be an independent risk factor of delayed STDT in all STEMI patients.

Cardiogenic shock (OR, 0.67; 95\% CI, 0.51 to $0.88 ; p$ $=0.003$ ) and utilisation of EMS (OR, 0.20; 95\% CI, 0.17 to $0.23 ; p<0.001$ ) were predictive factors for visiting a hospital within 60 minutes. Among patients with cardiogenic shock, old age (OR, 1.85; 95\% CI, 1.04 to 3.29; $p$ $=0.036)$, female (OR, 2.21; 95\% CI, 1.14 to $4.28 ; p=0.019)$, and diabetes (OR, 1.89; 95\% CI, 1.05 to $3.40 ; p=0.033$ ) were independent predictors of delayed STDT. In contrast, utilisation of EMS was related to STDT within 60 minutes (OR, 0.15 ; 95\% CI, 0.08 to $0.28 ; p<0.001$ ). 
Table 2. Medical treatments, procedural findings, and in-hospital outcomes

\begin{tabular}{|c|c|c|c|c|c|c|c|}
\hline \multirow[b]{2}{*}{ Characteristic } & \multirow{2}{*}{$\begin{array}{c}\text { Total } \\
(\mathrm{n}=4,874)\end{array}$} & \multicolumn{3}{|c|}{ All population $(\mathrm{n}=4,874)$} & \multicolumn{3}{|c|}{ Cardiogenic shock $(n=282)$} \\
\hline & & $\begin{array}{c}\mathrm{STDT} \leq 60 \\
\min (\mathrm{n}=1,386)\end{array}$ & $\begin{array}{c}\text { STDT }>60 \\
\min (n=3,488)\end{array}$ & $p$ value & $\begin{array}{l}\mathrm{STDT} \leq 60 \\
\min (\mathrm{n}=130)\end{array}$ & $\begin{array}{l}\mathrm{STDT}>60 \\
\min (\mathrm{n}=152)\end{array}$ & $p$ value \\
\hline Aspirin & $4,861(99 \cdot 7)$ & $1,379(99 \cdot 5)$ & $3,482(99.8)$ & 0.084 & $124(95 \cdot 4)$ & $149(98.0)$ & $0.35^{8}$ \\
\hline Clopidogrel & $3,074(63.1)$ & $834(60.2)$ & $2,240(64.2)$ & 0.009 & $70(53.8)$ & $85(55 \cdot 9)$ & 0.819 \\
\hline Ticagrelor & $1,043(21.4)$ & $325(23.4)$ & $718(20.6)$ & 0.031 & $25(19.2)$ & $25(16.4)$ & 0.650 \\
\hline Prasugrel & $607(12.5)$ & $182(13.1)$ & $425(12.2)$ & 0.393 & $15(11.5)$ & $10(6.6)$ & 0.211 \\
\hline Beta-blockers & $4,155(85.2)$ & $1,197(86.4)$ & $2,958(84.8)$ & 0.180 & $82(63.1)$ & $73(48.0)$ & 0.016 \\
\hline ACE inhibitors & $2,571(52.7)$ & $733(52.9)$ & $1,838(52.7)$ & 0.929 & $52(40.0)$ & $45(29.6)$ & 0.088 \\
\hline ARBs & $1,305(26.8)$ & $348(25.1)$ & $957(27.4)$ & 0.105 & $23(17.7)$ & $26(17 \cdot 1)$ & 1.000 \\
\hline Statins & $4,464(91.6)$ & $1,272(91.8)$ & $3,192(91.5)$ & 0.811 & $86(66.2)$ & $89(58.6)$ & 0.235 \\
\hline GP IIb/IIIa inhibitor & $1,076(22.1)$ & $309(22.3)$ & $767(22.0)$ & 0.847 & $42(32 \cdot 3)$ & $44(28.9)$ & 0.630 \\
\hline Multi-vessel diseasedz. & $2,265(46.5)$ & $638(46.0)$ & $1,627(46.6)$ & 0.722 & $76(58.5)$ & $95(62.5)$ & 0.569 \\
\hline LAD related diseasedz. & $2,456(50.4)$ & $713(51.4)$ & $1,743(50.0)$ & 0.371 & $40(30.8)$ & $55(36.2)$ & 0.405 \\
\hline Lesion type $\mathrm{B}_{1} / \mathrm{B}_{2}$ & $2,224(45 \cdot 6)$ & $612(44.2)$ & $1,612(46.2)$ & 0.204 & $58(44.6)$ & $62(40.8)$ & 0.598 \\
\hline Lesion type C & $2,609(53 \cdot 5)$ & $754(54 \cdot 4)$ & $1,855(53.2)$ & 0.461 & $69(53.1)$ & $89(58.6)$ & 0.422 \\
\hline Thrombus aspiration & $1,854(38.0)$ & $515(37.2)$ & $1,339(38.4)$ & 0.444 & $41(31.5)$ & $56(36.8)$ & 0.419 \\
\hline Using BMS & $131(2.7)$ & $38(2.7)$ & $93(2.7)$ & 0.961 & $9(6.9)$ & $11(7.2)$ & 1.000 \\
\hline IABP & $242(5.0)$ & $71(5 \cdot 1)$ & $171(4.9)$ & 0.806 & $27(20.8)$ & $47(30.9)$ & 0.073 \\
\hline ECMO & $75(1.5)$ & $27(1.9)$ & $48(1.4)$ & 0.182 & $18(13.8)$ & $19(12.5)$ & 0.875 \\
\hline $\begin{array}{l}\text { Complete } \\
\text { revascularisation }^{a}\end{array}$ & $4,809(98.7)$ & $1,369(98.8)$ & $3,440(98.6)$ & 0.568 & $123(94 \cdot 6)$ & $144(94 \cdot 7)$ & 0.748 \\
\hline Hospital death & $207(4.2)$ & $5^{8}(4.2)$ & $149(4 \cdot 3)$ & 0.954 & $33(25 \cdot 4)$ & $60(39.5)$ & 0.017 \\
\hline Cardiac death & $187(3.8)$ & $54(3 \cdot 9)$ & $133(3.8)$ & 0.957 & $32(24.6)$ & $55(36.2)$ & 0.049 \\
\hline Non-cardiac death & $20(0.4)$ & $4(0.3)$ & $16(0.5)$ & 0.555 & $1(0.8)$ & $5(3 \cdot 3)$ & 0.295 \\
\hline Newly developed HF & $172(3 \cdot 5)$ & $47(3.4)$ & $125(3.6)$ & 0.808 & $9(6.9)$ & $21(13.8)$ & 0.093 \\
\hline $\mathrm{VT} / \mathrm{VF}$ & $395(8.1)$ & $134(9.7)$ & $261(7 \cdot 5)$ & 0.014 & $41(31.5)$ & $54(35 \cdot 5)$ & 0.562 \\
\hline TIMI major bleeding & $100(2.1)$ & $30(2.2)$ & $70(2.0)$ & 0.812 & $15(11.5)$ & $14(9.2)$ & 0.656 \\
\hline TIMI minor bleeding & $150(3.1)$ & $38(2.7)$ & $112(3.2)$ & 0.445 & $9(6.9)$ & $10(6.6)$ & 1.000 \\
\hline
\end{tabular}

Values are presented as number (\%).

STDT, symptom-to-door time; ACE, angiotensin-converting enzyme; ARB, angiotensin II receptor blocker; GP, glycoprotein; $\mathrm{dz}$, disease; LAD, left anterior descending artery; BMS, bare metal stent; IABP, intra-aortic balloon pump; ECMO, extracorporeal membrane oxygenation; HF, heart failure; VT/VF, ventricular tachycardia/ventricular fibrillation; TIMI, thrombolysis in myocardial infarction.

${ }^{a}$ Angiographic complete revascularisation was defined as minimum stenosis diameter reduction to less than $20 \%$.

\section{Clinical outcomes and predictors of non-utilisation of EMS}

Significant differences in baseline characteristics were observed for certain types of hospital visits (Table 3). After the index procedure for treating STEMI, the clinical outcomes according to the type of hospital visit were illustrated in Fig. 4. Among the all study population, the mortality rate of the EMS group was similar to that of the
non-PCI centre group (Fig. 4A), although the incidence of cardiogenic shock was higher in the EMS group than in other groups. In a subgroup analysis of patients with cardiogenic shock, utilising EMS was associated with more favourable outcomes, as compared to the non-PCI centre group (Fig. 4B). This trend continued during the 3-year follow-up period, in terms of the cumulative incidence of MACE (Fig. 4C and $4 \mathrm{D}$ ). 


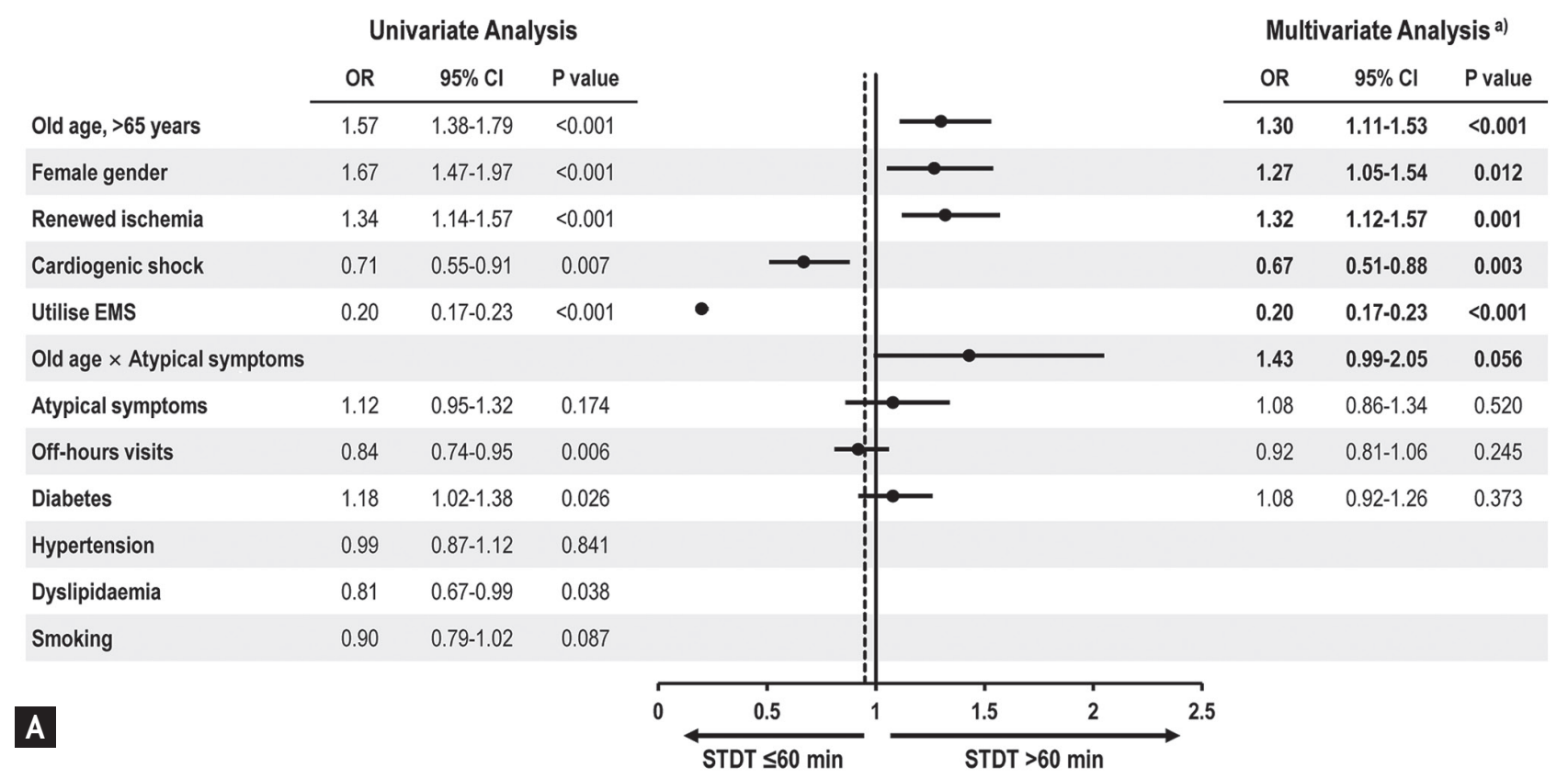

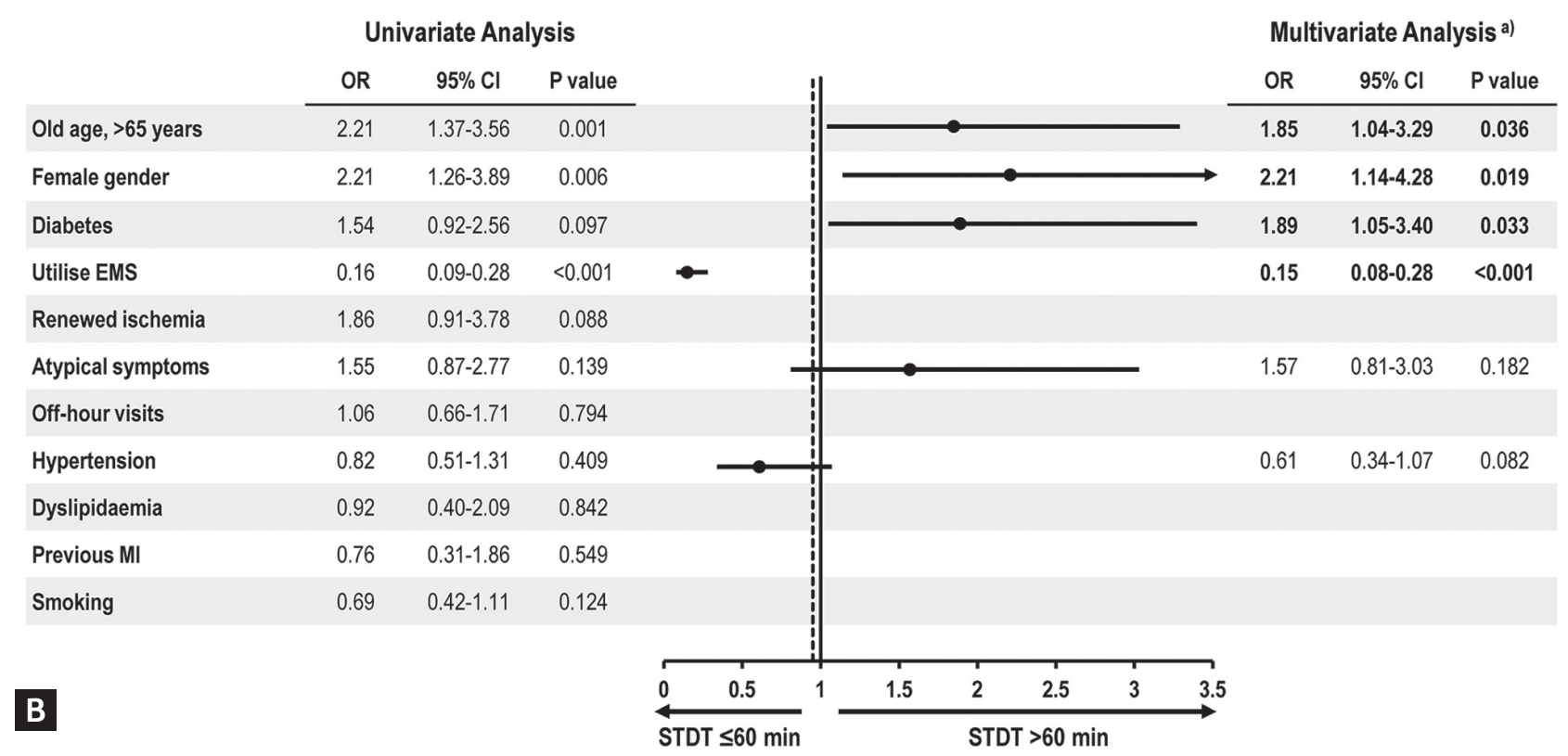

Figure 3. Predictors for pre-hospital delay (symptom-to-door time [STDT] > 6o minutes). The results of uni- and multivariate analyses in (A) all patients and (B) patients with cardiogenic shock were outlined. Only utilising emergency medical services (EMS) was an independent predictor of shortening pre-hospital delay, regardless of haemodynamics. OR, odds ratio; CI, confidence interval; MI, myocardial infarction. ${ }^{a}$ Multivariate analysis was performed using backward elimination with variables that showed a $p<0.1$ in univariate analysis.

To identify risk factors associated with non-utilisation of EMS, several factors were included as candidate variables (Supplementary Table 1). Fig. 5 shows the results of univariate and multivariate logistic regression analy- ses to identify predictors of non-utilisation of EMS. The final multivariate logistic regression analysis showed moderate to good levels of prediction (c-statistics, 0.715). Female gender was associated with non-utilisation of 


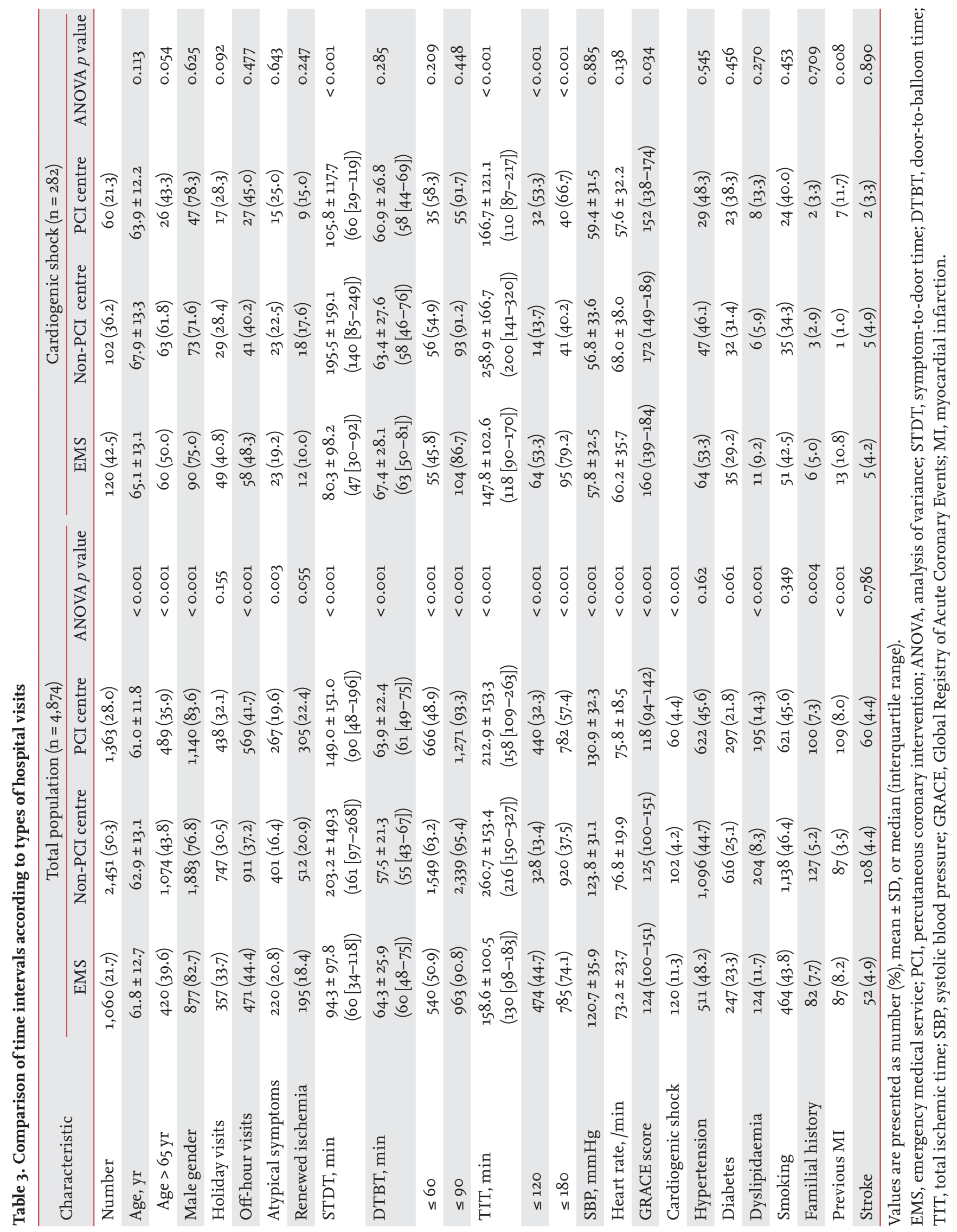



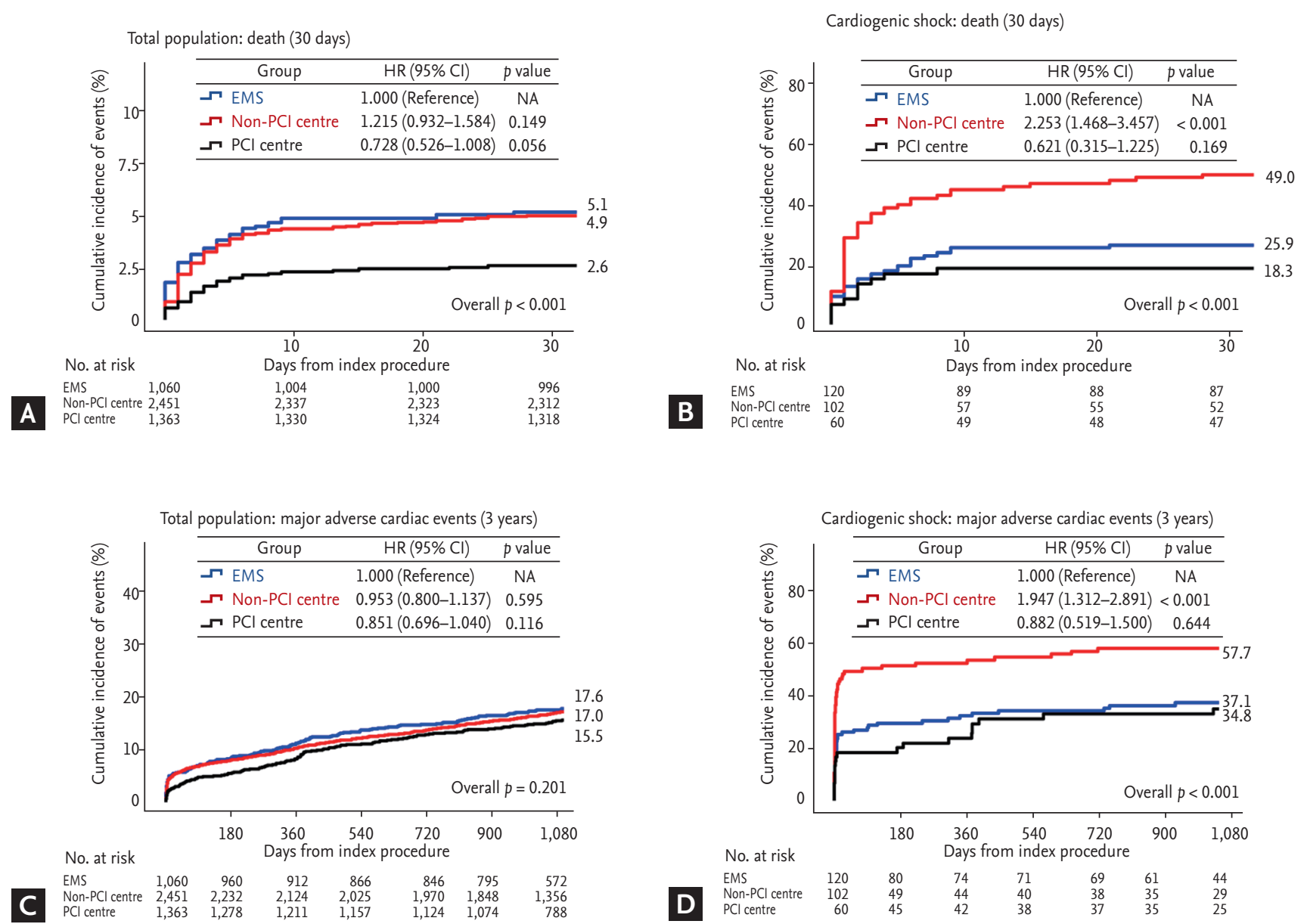

Figure 4. Clinical outcomes according to type of hospital visit (emergency medical services [EMS] vs. non-percutaneous coronary intervention [PCI] centre vs. PCI centre). Survival analyses using Kaplan-Meier curves with cumulative hazards of(A) allcause death of all patients and (B) patients with cardiogenic shock within 30 days; and (C) major adverse cardiac events of all patients and (D) patients with cardiogenic shock at 3 years. HR, hazard ratio; CI, confidence interval; NA, not available.

EMS (OR, 1.25; 95\% CI, 1.03 to 1.51; $p=0.022)$. Cardiogenic shock (OR, $0.34 ; 95 \% \mathrm{CI}, 0.27$ to $0.44 ; p<0.001$ ), previous MI (OR, $0.61 ; 95 \% \mathrm{CI}, 0.47$ to 0.80 ; $p<0.001$ ), familial history of IHD (OR, $0.75 ; 95 \% \mathrm{CI}, 0.57$ to $0.98 ; p=0.033$ ), and off-hour visits (OR, $0.81 ; 95 \% \mathrm{CI}, 0.71$ to $0.93 ; p=0.003$ ) were independent predictors of the utilisation of EMS.

\section{DISCUSSION}

The present study evaluated factors predicting a pre-hospital delay by evaluating the STDT and utilisation of EMS among STEMI patients who underwent PPCI. The major findings were as follows. First, improved STDT was associated with an overall improvement in TIT.
Second, old age, female gender, and renewed ischemia were independent factors for predicting delayed STDT. Third, patients who utilised EMS were more likely to arrive at the hospital within 60 minutes, regardless of the presence of cardiogenic shock. Fourth, female gender was an independent factor predicting non-utilisation of EMS, whereas cardiogenic shock, previous MI, familial history of IHD, and off-hour visits were associated with non-utilisation of EMS.

Minimising TIT is a major determinant of myocardial salvage, as the prolonged duration of ischemia is related to myocardial necrosis, as evidenced by contrast-enhanced magnetic resonance [13]. The median DTBT observed in this study was close to the target of 60 to 90 minutes between symptom onset and arrival at the 


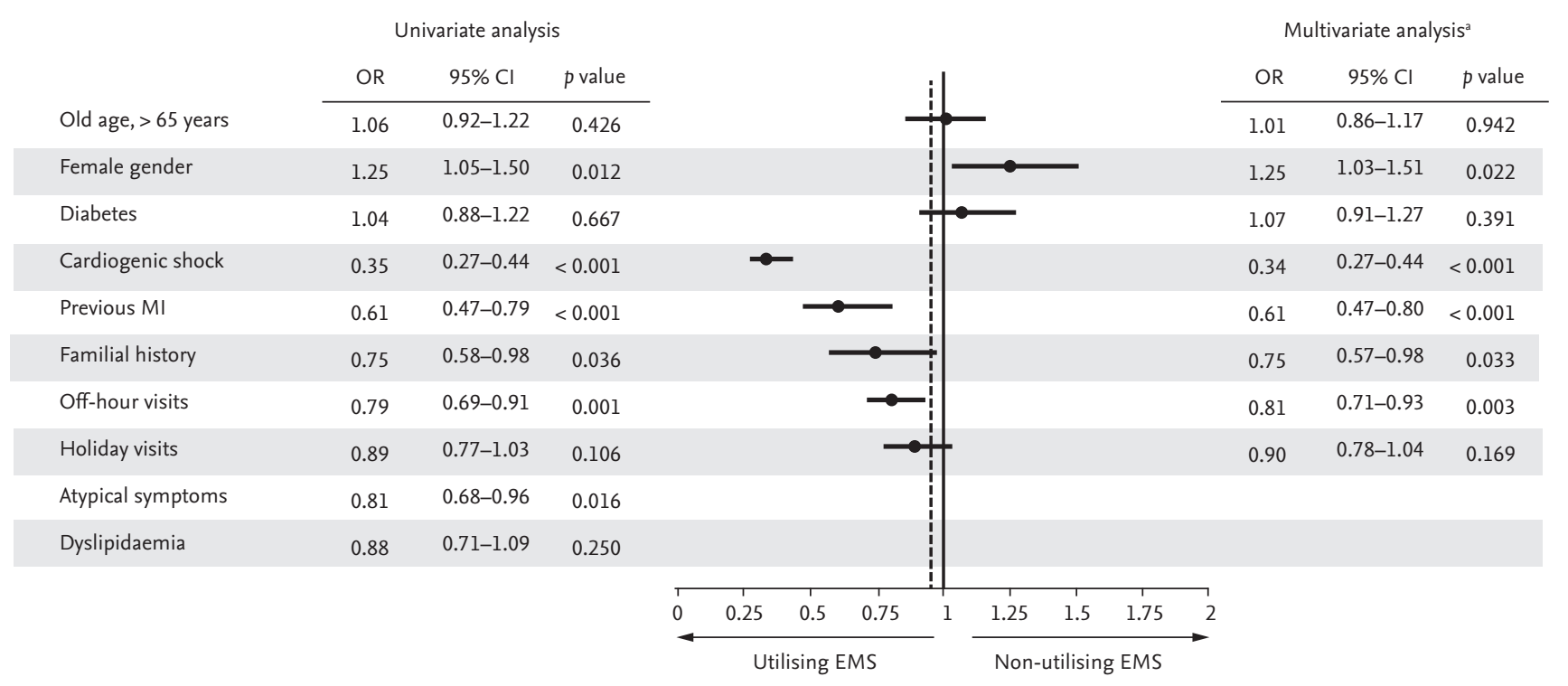

Figure 5. Predictors for non-utilisation of emergency medical services (EMS). Univariate and multivariate analyses revealed predictors for non-utilisation of EMS. OR, odds ratio; CI, confidence interval; MI, myocardial infarction. ${ }^{\mathrm{a}}$ Multivariate analysis was performed using backward elimination with variables that showed a $p<0.1$ in univariate analysis.

hospital, suggested by current guidelines [12,14]. However, adherence to these guidelines provided no improvement in pre-hospital delay, and took more time than expected. The median STDT of the total study population was $120 \mathrm{~min}$, consistent with previous studies which showed median STDT ranges of 120 to 171 minutes in the United States, Japan, and Korea [15-17]. Since TIT was more closely related to STDT than to DTBT, only a few patients $(25.5 \%)$ achieved the goal of TIT within 120 minutes and $51 \%$ within 180 minutes for myocardial salvage, as suggested by previous studies [18,19].

After stratification by STDT with a cut-off of 60 minutes, approximately one-third of all patients were categorised into the STDT $\leq 60$ minutes group. The median STDT of this group was 39 minutes (29 to 56 ), and $77.8 \%$ achieved TIT within 120 minutes. In contrast, only $4.7 \%$ of patients in the STDT > 6o minutes group achieved TIT within 120 minutes, with a median STDT of 169 minutes (111 to 273). These time trends were similar among patients with cardiogenic shock. Consequently, to improve TIT in real-world clinical practice, reducing pre-hospital delays may be more important than reducing DTBT. A target STDT of 60 minutes would therefore be reasonable to achieve TIT within 120 to 180 minutes.

STDT consists of two main components: patient decision time and transport time [20]. Previous studies have focused on identifying the clinical, socioeconomic, or regional factors that cause a delayed STDT among STEMI patients [15,20-28]. Those risk factors were female gender [20-25], older age [21-23,26], diabetes [21-24], and atypical symptoms $[22,25-27]$. Other predictors might be night time presentation [15,23], longer decision time [20,25], and non-utilisation EMS [15,22,28,29]. Although public campaigns and educational programs targeting those factors have been implemented, there is little evidence that they have successfully reduced treatment delays [30]. The data presented here suggest that recent efforts in Korea have not been effective for reducing STDT and TIT. This phenomenon may be the result of a wider range target for STDT of 2 to 3 hours, compared to the much shorter duration shown here. Moreover, it is doubtful that STDT could be reduced by educational programs aimed at the general public.

Based on studies using preventive interventions, which revealed that high-risk prevention strategies could increase the efficiency of public medical education [31,32], we sought to identify crucial factors related to a delay STDT > 60 minutes among STEMI patients. We confirmed that old age, female gender, and renewed ischemia were independent factors predictive of delayed STDT. Both older age and female gender have previously been associated with atypical symptoms, and 
have been shown to strongly affect STDT [25,26]; hence, we included the interaction terms in the multivariate analyses. Because patients who presented with cardiogenic shock were likely to visit the hospital earlier, an additional subgroup analysis for cardiogenic shock was performed. Among patients presenting with cardiogenic shock, old age, female gender, and diabetes were identified as independent risk factors associated with STDT $>60$ minutes.

Although the socioeconomic status of aged and female patients would have affected STDT in a similar way to that found in previous studies examining decision time, the current results suggest that other distinguishing factors may need to be addressed. Before the index presentation of STEMI, some patients (20.8\%) had already experienced ischemic symptoms. These experiences may have influenced STDT because STEMI symptoms could be misinterpreted as "simple" symptoms. The renewed ischemia may interfere with the decision-making process and result in a failure to achieve the "golden hour" for myocardial salvage. Furthermore, the association between old age and atypical symptoms was significantly related to delay of STDT, suggesting that we should focus on those who present with both variables simultaneously. Thus, it is necessary to modify public education programs to ensure that patients with suspected angina and/or older patients with atypical symptoms visit their healthcare providers.

Jager et al. [24] reported that one of the strongest predictors of shorter STDT is cardiogenic shock. Patients with cardiogenic shock usually present earlier because the warning signs of tissue hypoperfusion and decompensated heart failure, such as impaired consciousness, hypotension, and desaturation, encourage patients to visit the hospital. Moreover, delay of reperfusion is a strong predictor of adverse outcomes among patients with cardiogenic shock. Any factors delaying prompt treatment should therefore be identified and removed [33]. Our study confirmed that cardiogenic shock was related to timely arrival at the hospital. Among patients with cardiogenic shock, old age, female gender, and diabetes remained independent predictors for delayed STDT > 60 minutes.

Kim et al. [20] reported that the median decision time to seek medical assistance is 20 to 25 minutes in Korea. Based on this finding, transport time, which accounts for the remainder of STDT, might be calculated indirectly as > 6o minutes. If patients initially utilise EMS to avoid traffic congestion in overcrowded urban areas, the transport time and STDT may be reduced. Linear regression curves classified by types of hospital visits (Supplementary Fig. 2) found that utilisation of EMS was related to shorter STDT; conversely, direct visits to PCI centres or transfer from non-PCI centres were related to longer STDT. However, the data presented here showed that direct visits to PCI centres (28\%) or transfers from non-PCI centres (50.3\%) were more frequent, and utilisation of EMS (21.7\%) was less frequent than that seen in previous studies conducted in Western countries $[29,34,35]$. Despite frequencies similar to those of other Asian countries [36,37], a significant need to identify predictors of non-utilisation of EMS among STEMI patients remains. Although certain clinical variables have been linked to non-use of EMS services, these results remain controversial [36-39]. In accordance with other studies, old age was not shown to be an independent factor for non-utilisation of EMS. In contrast, female gender was associated with non-utilisation of EMS, whereas cardiogenic shock, previous MI, familial history of IHD, and off-hour visits were all related to use of EMS.

\section{Study limitations}

The current study has several limitations that should be considered. First, it was a retrospective analysis of an observational cohort, which has some potential for selection and confounding bias. It is difficult to evaluate the clinical impact of STDT or EMS using a randomised prospective clinical trial due to methodological and ethical problems. This study may, therefore, represent one of the largest studies to date for identifying predictors in real-world practice. Second, we could not fully explain the mechanisms by which the predictors identified in this analysis influence STDT or EMS. Socioeconomic status, education level, and familial environment may all be related to a delay in STDT; however, we were not able to evaluate those effects in this analysis. The impact of those variables on STDT has been established in previous studies; thus, the limitation of the current study is somewhat reduced. In addition, there were no data on geographical characteristics, which may be associated with STDT or the accessibility of EMS. Although this study sought to identify patient factors for reducing 
STDT using a high-risk prevention strategy, educational programs should also pay close attention to demographic characteristics. Third, our data will require continuous updating to reflect recent temporal trends. We expect to overcome these limitations by continuing our analysis of the KAMIR-NIH data, with new results from the 5-year follow-up study helping to overcome these limitations.

In conclusion, pre-hospital delay, as defined by STDT, may be an important target for reducing TIT. To improve TIT to a maximum of 120 to 180 minutes, we suggest that STDT be reduced to $<60$ minutes. Several clinical risk factors including old age, female gender, and renewed ischemia were all identified as independent risk factors for delayed STDT. Utilisation of EMS was the only modifiable predictor related to improvements in pre-hospital delay. In contrast, female gender was identified as an independent factor associated with non-utilisation of EMS, whereas cardiogenic shock, previous MI, familial history of IHD, and off-hour visits were all independent factors related to the promotion of EMS. Identification of risk factors associated with delayed STDT or non-utilisation of EMS among STEMI patients is necessary, therefore, to establish high-risk prevention strategies to reduce TIT in real-world practice. Healthcare providers should consider intensive educational programs focusing on improving STDT or encouraging use of EMS to reduce TIT.

\section{KEY MESSAGE}

1. Pre-hospital delay, as defined by symptom-todoor time (STDT), is an important target for reducing total ischemic time (TIT).

2. Old age, female gender, and renewed ischemia were independent factors for predicting delayed STDT.

3. Utilisation of emergency medical services (EMS) was the only modifiable predictor for reducing STDT. Therefore, educational programs focusing on the use of EMS should be considered to reduce TIT.

\section{Conflict of interest}

No potential conflict of interest relevant to this article was reported.

\section{Acknowledgments}

The authors thank the clinical investigators of the Korea Acute Myocardial Infarction Registry who contributed their time and effort to this study. This research was supported by a grant from the Research of Korea Centers for Disease Control and Prevention (2016-ER6304o1). This study was supported by research fund from Chosun University, 2016.

\section{REFERENCES}

1. De Luca G, Suryapranata H, Ottervanger JP, Antman EM. Time delay to treatment and mortality in primary angioplasty for acute myocardial infarction: every minute of delay counts. Circulation 2004;109:1223-1225.

2. De Luca G, van 't Hof AW, de Boer MJ, et al. Time-totreatment significantly affects the extent of ST-segment resolution and myocardial blush in patients with acute myocardial infarction treated by primary angioplasty. Eur Heart J 2004;25:1009-1013.

3. Francone M, Bucciarelli-Ducci C, Carbone I, et al. Impact of primary coronary angioplasty delay on myocardial salvage, infarct size, and microvascular damage in patients with ST-segment elevation myocardial infarction: insight from cardiovascular magnetic resonance. J Am Coll Cardiol 2009;54:2145-2153.

4. Kim HK, Jeong MH, Ahn Y, et al. Relationship between time to treatment and mortality among patients undergoing primary percutaneous coronary intervention according to Korea Acute Myocardial Infarction Registry. J Cardiol 2017;69:377-382.

5. McGinn AP, Rosamond WD, Goff DC Jr, Taylor HA, Miles JS, Chambless L. Trends in prehospital delay time and use of emergency medical services for acute myocardial infarction: experience in 4 US communities from 19872000. Am Heart J 2005;150:392-400.

6. Jollis JG, Al-Khalidi HR, Roettig ML, et al. Impact of regionalization of ST-segment-elevation myocardial infarction care on treatment times and outcomes for emergency medical services-transported patients presenting to hospitals with percutaneous coronary intervention. Mission: Lifeline Accelerator-2. Circulation 2018;137:376387. 
7. Kim JH, Chae SC, Oh DJ, et al. Multicenter cohort study of acute myocardial infarction in Korea: interim analysis of the Korea Acute Myocardial Infarction Registry-National Institutes of Health Registry. Circ J 2016;80:14271436.

8. Thygesen K, Alpert JS, Jaffe AS, et al. Third universal definition of myocardial infarction. Eur Heart J 2012;33:25512567.

9. Thiele H, Akin I, Sandri M, et al. PCI strategies in patients with acute myocardial infarction and cardiogenic shock. N Engl J Med 2017;377:2419-2432.

10. Kushner FG, Hand M, Smith SC Jr, et al. 2009 Focused updates: ACC/AHA guidelines for the management of patients with ST-elevation myocardial infarction (updating the 2004 guideline and 2007 focused update) and ACC/ AHA/SCAI guidelines on percutaneous coronary intervention (updating the 2005 guideline and 2007 focused update): a report of the American College of Cardiology Foundation/American Heart Association Task Force on Practice Guidelines. Circulation 2009;120:2271-2306.

11. Task Force on the management of ST-segment elevation acute myocardial infarction of the European Society of Cardiology (ESC), Steg PG, James SK, et al. ESC guidelines for the management of acute myocardial infarction in patients presenting with ST-segment elevation. Eur Heart J 2012;33:2569-2619.

12. O'Gara PT, Kushner FG, Ascheim DD, et al. 2013 ACCF/ AHA guideline for the management of ST-elevation myocardial infarction: a report of the American College of Cardiology Foundation/American Heart Association Task Force on Practice Guidelines. J Am Coll Cardiol 2013;61:e78-e140.

13. Tarantini G, Cacciavillani L, Corbetti F, et al. Duration of ischemia is a major determinant of transmurality and severe microvascular obstruction after primary angioplasty: a study performed with contrast-enhanced magnetic resonance. J Am Coll Cardiol 2005;46:1229-1235.

14. Ibanez B, James S, Agewall S, et al. 2017 ESC guidelines for the management of acute myocardial infarction in patients presenting with ST-segment elevation: the task force for the management of acute myocardial infarction in patients presenting with ST-segment elevation of the European Society of Cardiology (ESC). Eur Heart J 2018;39:119-177.

15. Park YH, Kang GH, Song BG, et al. Factors related to prehospital time delay in acute ST-segment elevation myo- cardial infarction. J Korean Med Sci 2012;27:864-869.

16. Hafiz AM, Naidu SS, DeLeon J, et al. Impact of first contact on symptom onset-to-door time in patients presenting for primary percutaneous coronary intervention. Am J Emerg Med 2013;31:922-927.

17. Fujii T, Masuda N, Suzuki T, et al. Impact of transport pathways on the time from symptom onset of ST-segment elevation myocardial infarction to door of coronary intervention facility. J Cardiol 2014;64:11-18.

18. Boersma E, Maas AC, Deckers JW, Simoons ML. Early thrombolytic treatment in acute myocardial infarction: reappraisal of the golden hour. Lancet 1996;348:771-775.

19. Eitel I, Desch S, Fuernau G, et al. Prognostic significance and determinants of myocardial salvage assessed by cardiovascular magnetic resonance in acute reperfused myocardial infarction. J Am Coll Cardiol 2010;55:2470-2479.

20. Kim HS, Lee KS, Eun SJ, et al. Gender differences in factors related to prehospital delay in patients with ST-segment elevation myocardial infarction. Yonsei Med J 2017;58:710-719.

21. Gibler WB, Armstrong PW, Ohman EM, et al. Persistence of delays in presentation and treatment for patients with acute myocardial infarction: the GUSTO-I and GUSTO-III experience. Ann Emerg Med 2002;39:123-130.

22. Goldberg RJ, Steg PG, Sadiq I, et al. Extent of, and factors associated with, delay to hospital presentation in patients with acute coronary disease (the GRACE registry). Am J Cardiol 2002;89:791-796.

23. Nielsen CG, Laut KG, Jensen LO, Ravkilde J, Terkelsen CJ, Kristensen SD. Patient delay in patients with ST-elevation myocardial infarction: time patterns and predictors for a prolonged delay. Eur Heart J Acute Cardiovasc Care 2017;6:583-591.

24. Jager B, Farhan S, Rohla M, et al. Clinical predictors of patient related delay in the VIENNA ST-elevation myocardial infarction network and impact on long-term mortality. Eur Heart J Acute Cardiovasc Care 2017;6:254261.

25. Sederholm Lawesson S, Isaksson RM, Ericsson M, Angerud K, Thylen I; SymTime Study Group. Gender disparities in first medical contact and delay in ST-elevation myocardial infarction: a prospective multicentre Swedish survey study. BMJ Open 2018;8:e020211.

26. Grossman SA, Brown DF, Chang Y, et al. Predictors of delay in presentation to the ED in patients with suspected acute coronary syndromes. Am J Emerg Med 2003;21:425- 
428.

27. Thuresson M, Jarlov MB, Lindahl B, Svensson L, Zedigh C, Herlitz J. Thoughts, actions, and factors associated with prehospital delay in patients with acute coronary syndrome. Heart Lung 2007;36:398-409.

28. AlHabib KF, Sulaiman K, Al Suwaidi J, et al. Patient and system-related delays of emergency medical services use in acute ST-elevation myocardial infarction: results from the third Gulf Registry of Acute Coronary Events (Gulf RACE-3Ps). PLoS One 2016;11:e0147385.

29. McKee G, Mooney M, O'Donnell S, O’Brien F, Biddle MJ, Moser DK. Multivariate analysis of predictors of pre-hospital delay in acute coronary syndrome. Int J Cardiol 2013;168:2706-2713.

30. Kainth A, Hewitt A, Sowden A, et al. Systematic review of interventions to reduce delay in patients with suspected heart attack. Emerg Med J 2004;21:506-508.

31. Kelly JA, Murphy DA, Sikkema KJ, et al. Randomised, controlled, community-level HIV-prevention intervention for sexual-risk behaviour among homosexual men in US cities. Community HIV Prevention Research Collaborative. Lancet 1997;350:1500-1505.

32. Merzel C, D'Afflitti J. Reconsidering community-based health promotion: promise, performance, and potential. Am J Public Health 2003;93:557-574.

33. Scholz KH, Maier SKG, Maier LS, et al. Impact of treatment delay on mortality in ST-segment elevation myocardial infarction (STEMI) patients presenting with and without haemodynamic instability: results from the German prospective, multicentre FITT-STEMI trial. Eur Heart J 2018;39:1065-1074.

34. Canto JG, Zalenski RJ, Ornato JP, et al. Use of emergency medical services in acute myocardial infarction and subsequent quality of care: observations from the National Registry of Myocardial Infarction 2. Circulation 2002;106:3018-3023.

35. Kerr D, Holden D, Smith J, Kelly AM, Bunker S. Predictors of ambulance use in patients with acute myocardial infarction in Australia. Emerg Med J 2006;23:948-952.

36. Fares S, Zubaid M, Al-Mahmeed W, et al. Utilization of emergency medical services by patients with acute coronary syndromes in the Arab Gulf States. J Emerg Med 2011;41:310-316.

37. Ma J, Wang J, Zheng W, et al. Usage of ambulance transport and influencing factors in acute coronary syndrome: a cross-sectional study at a tertiary centre in China. BMJ Open 2017;7:e015809.

38. Brown AL, Mann NC, Daya M, et al. Demographic, belief, and situational factors influencing the decision to utilize emergency medical services among chest pain patients. Rapid Early Action for Coronary Treatment (REACT) study. Circulation 2000;102:173-178.

39. Thuresson M, Jarlov MB, Lindahl B, Svensson L, Zedigh C, Herlitz J. Factors that influence the use of ambulance in acute coronary syndrome. Am Heart J 2008;156:170-176. 


\section{Supplementary Table 1. Candidate variables for model construction}

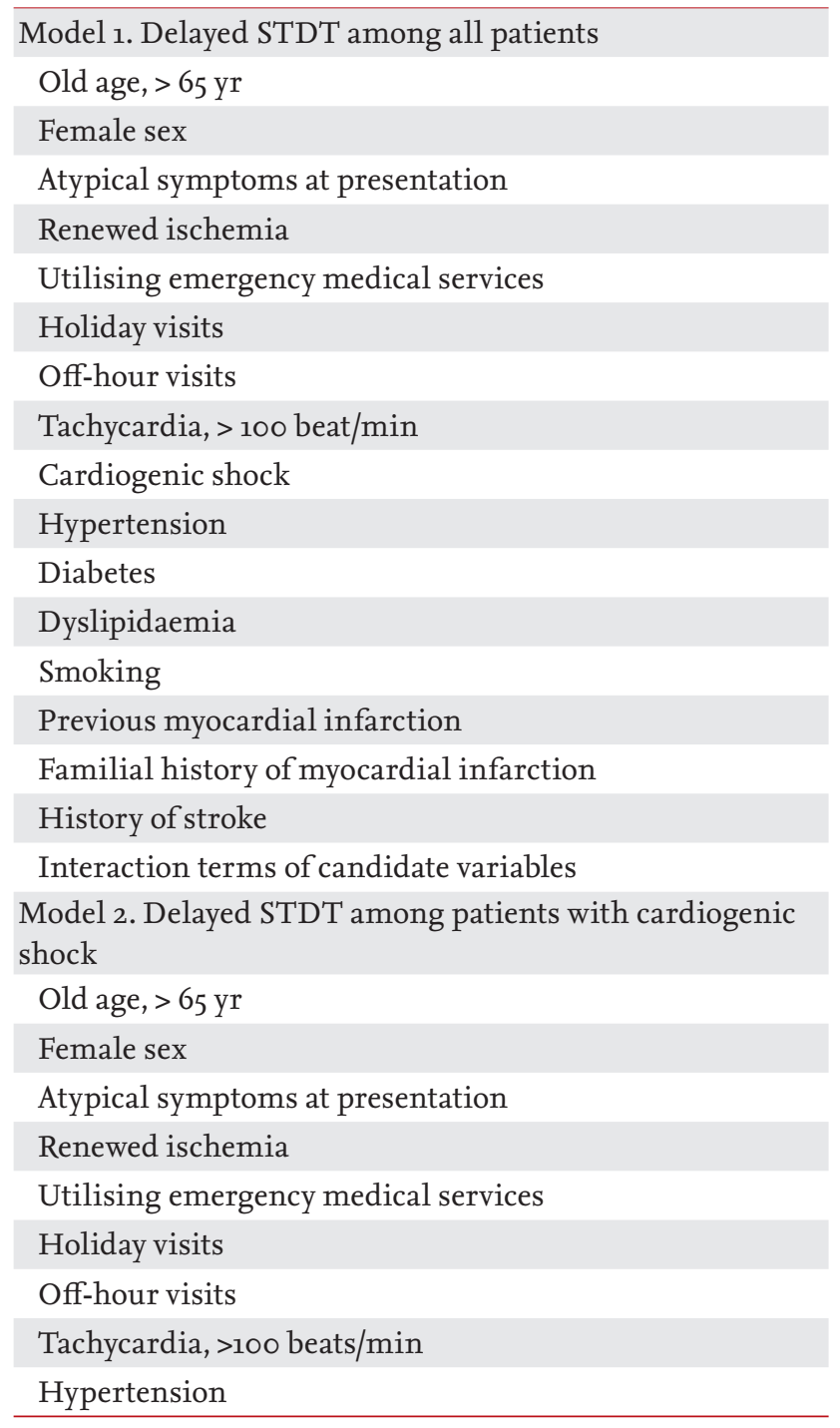

\begin{tabular}{l}
\hline Diabetes \\
Dyslipidaemia \\
Smoking \\
Previous myocardial infarction \\
Familial history of myocardial infarction \\
\hline History of stroke \\
\hline Interaction terms of candidate variables \\
\hline Model 3. Non-utilizing EMS \\
\hline Old age, $>$ 65 yr \\
\hline Female sex \\
\hline Atypical symptoms at presentation \\
\hline Renewed ischemia \\
Holiday visits \\
\hline Off-hour visits \\
\hline Tachycardia, > 1oo beat/min \\
\hline Cardiogenic shock \\
\hline Hypertension \\
\hline Diabetes \\
\hline Dyslipidaemia \\
\hline Smoking \\
\hline Previous myocardial infarction \\
\hline Familial history of myocardial infarction \\
\hline Interaction terms of candidate variables \\
\hline
\end{tabular}

Variables were considered for model construction if they demonstrated associations with pre-hospital delay or utilising emergency medical services in previously published literature, even though their $p$ values were less than 0.1 in the univariate analyses.

STDT, symptom-to-door time; EMS, emergency medical services. 

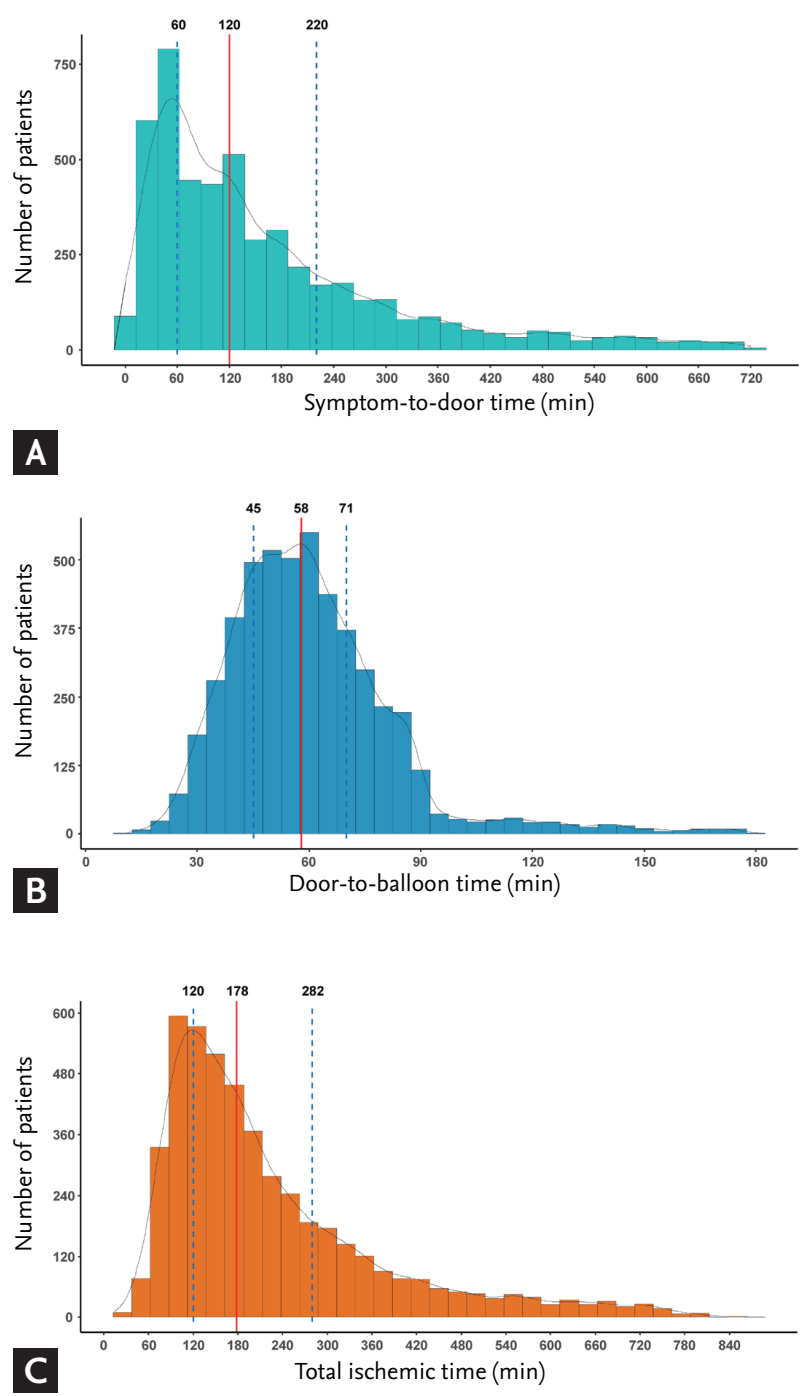

Supplementary Figure 1. Histograms and distribution curves according to timer intervals. (A) Distribution of symptom-to-door time. (B) Distribution of door-to-balloon time. (C) Distribution total ischemic time. 


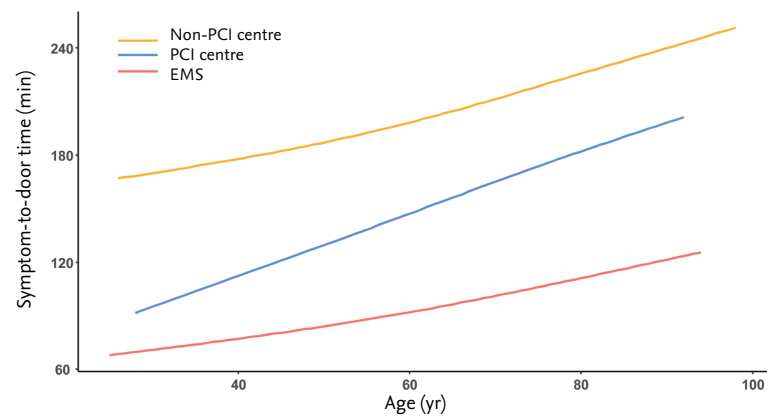

Supplementary Figure 2. Trends of symptom-to-door time (STDT) according to age and type of hospital visits. PCI, percutaneous coronary interventionfi; EMS, emergency medical services. 